\title{
Questionário de Condições de Trabalho para Servidores Universitários
}

\author{
Livia de Oliveira Borges ${ }^{1}$ (D), Geraldo Majela Garcia Primo (D) \\ Universidade Federal de Minas Gerais, Belo Horizonte-MG, Brasil \\ Sabrina Cavalcanti Barros (D) \\ Universidade Federal do Rio Grande do Norte, Santa Cruz-RN, Brasil \\ Silvino Paulino dos Santos Neto(D) \\ Centro Mineiro de Ensino Superior Promove, Belo Horizonte-MG, Brasil \\ Camila Teixeira Heleno (D) \\ Universidade Federal dos Vales de Jequitinhonha e Mucuri-MG, Brasil \\ Georgina Maria Véras Motta \\ Universidade Federal do Minas Gerais, Belo Horizonte-MG, Brasil
}

\section{RESUMO}

Adotamos o conceito de condições de trabalho abrangendo aspectos do conteúdo e do entorno do trabalho. Entretanto, havia a demanda de explorar as suas especificidades para servidores de universidades federais, visando uma melhor aproximação da realidade e, simultaneamente, explorar sua variabilidade histórica como esperado teoricamente. A pesquisa, então, almejou desenvolver uma versão do Questionário das Condições de Trabalho (QCT) para os servidores da UFMG e aperfeiçoá-lo. Modificamos o QCT adaptando itens e introduzindo outros; exploramos as estruturas fatoriais e avaliamos seu aperfeiçoamento psicométrico. Aplicamos questionários estruturados em 1.060 participantes. Por meio de análise fatorial exploratória, encontramos uma estrutura empírica com fatores de segunda e primeira ordem. Os de segunda ordem divergem das categorias teóricas aplicadas em pesquisas anteriores. Esses fatores e os primários (p. ex., Processo Burocrático e Participação) refletiram a percepção dos servidores acerca da realidade vivencial. A nova versão do QCT pode ser útil em diagnósticos ocupacionais.

Palavras-chave: Condições de Trabalho; Servidor Público; Universidades; Análises Fatoriais; validade de construto.

\section{ABSTRACT - Working Conditions Questionnaire for public workers in the university}

We adopted the concept of working conditions covering the content and surrounding aspects of work. However, there is a need to explore its specificities for public workers in federal universities with the intention of constructing a better approximation to reality, and, simultaneously, exploring their historical variability as theoretical expected. Accordingly, this study aimed to develop a version of the Working Conditions Questionnaires (WCQ) for UFMG's public workers and improve it. We changed the WCQ, adapting items and creating others; we explored the factorial structures; and evaluated their psychometric properties. We applied the structured questionnaire with 1,060 participants. Through exploratory factor analysis, we found an empirical structure with first and second order factors. The second order factors differed from the theoretical categories applied in previous studies. The factors found (e.g., Bureaucratic Process and Participation) reflected the public workers perception about the reality experienced. The new version of the WCQ can be useful in occupational diagnostics.

Keywords: Working Conditions; Public Worker; Universities; Factor Analysis; Construct Validity.

\section{RESUMEN - Cuestionario de Condiciones Laborales para Servidores Universitarios}

Adoptamos el concepto de condiciones laborales abarcando aspectos del contenido y del entorno del trabajo. Sin embargo, hubo una demanda de explorar sus especificidades para empleados de universidades federales, mirando una mejor aproximación de la realidad y, simultáneamente, explorar su variabilidad histórica como teóricamente se esperaba. La investigación, entonces, tuvo como objetivo desarrollar una versión del Cuestionario de Condiciones Laborales para los servidores de la UFMG, y mejorarla. Se modificó el QCT adaptando ítems y creando otros; se exploró las estructuras factoriales; y se evaluó su perfeccionamiento psicométrico. Los cuestionarios estructurados fueron contestados por 1.060 participantes. Por intermedio del análisis factorial exploratorio, se encontró una estructura empírica con factores de primer y segundo orden. Los de según orden difieren de las categorías teóricas de investigaciones previas. Estos factores y los primarios (por ejemplo, Procesos Burocráticos y Participación) reflejaron la percepción de los servidores acerca de la realidad vivencial. La nueva versión del QCT puede ser útil en diagnósticos ocupacionales.

Palabras claves: Condiciones Laborales; Servidor Público; Universidades; Análisis Factorial; Validez de Constructo.

Pesquisas (p. ex., García López, 2017; Ordaz Castillo \& Ronda-Pérez, 2015; Souza, 2014) têm sido realizadas sobre condições de trabalho e, entre elas, há aquelas sobre o Questionário de Condições de Trabalho 
(QCT), para operários da construção de edificações, para docentes de centro universitário particular e profissionais de saúde de um hospital universitário (Borges et al., 2013), bem como para trabalhadores do saneamento básico (Tertuliano \& Borges, 2019). De um lado, os resultados corroboraram haver diferentes fatores, conforme a expectativa de que as condições concretas de trabalho variam por ocupações, por instrução e forma de percepção dos trabalhadores. De outro lado, dos 16 fatores para docentes e profissionais de saúde, sete com coeficientes alfa de Cronbach entre 0,58 a 0,75 (Borges et al., 2013) sublinharam a demanda de aperfeiçoamento do QCT.

Apesar dessas deficiências de medida, para os profissionais de saúde, sete fatores das condições de trabalho diferenciaram (ANOVA) significativamente perfis de saúde psíquica (Costa et al., 2015). Para os docentes do centro universitário já referidos, onze fatores mostraram-se preditores (path analysis) de comprometimento organizacional e com a carreira (Souza, 2014). Essas variâncias conjuntas das condições de trabalho com outros fenômenos indicaram validade de critério (Pasquali, 2003), que, contrastando às fragilidades antes referidas acerca do QCT, justifica buscar seu aperfeiçoamento, superar limitações e explorar seu caráter contingencial às ocupações.

Do ponto de vista da escolha de um segmento ocupacional, consideramos que pesquisas (p. ex., Costa et al., 2015; Forattini \& Lucena, 2015; Geraldini \& Bicalho, 2016; Pizzio \& Klein, 2015; Ramos \& Macêdo, 2018; Ruza \& Silva, 2016; Santos et al., 2016; Tavares et al., 2012) têm evidenciado a prevalência de alterações de saúde em servidores públicos e associado às condições de trabalho. Maia et al. (2015) argumentaram que investir em melhorias de condições de trabalho, além dos impactos em qualidade de vida do trabalhador, pode ampliar a efetividade organizacional. Com a intenção de contribuir com instrumentos úteis a universidades federais em diagnósticos de condições de trabalho, planejamos pesquisa com o objetivo geral de desenvolver uma versão do QCT específica para os servidores (docentes e funcionários técnico-administrativos, TAEs) na UFMG e avaliar suas evidências de validade.

\section{Quadro Teórico e Objetivos Específicos}

Como alguns autores (p. ex., Álvaro \& Garrido, 2006; Blanch, 2003; García López, 2017; Ordaz Castillo \& Ronda-Pérez, 2015; Pietro et al., 2009; Ramos et al., 2002), filiamos-nos a conceituação de condições de trabalho proposta incialmente por Pietro (1994), segundo quem elas abrangem o entorno e o conteúdo do trabalho, de forma que influencia, diretamente, a experiência e as relações de trabalho. Blanch (2003), Álvaro e Garrido (2006) e Ramos et al. (2002) propuseram taxonomias e sublinharam as possíveis variações por ocupações e por fatores conjunturais, ou seja, contrapuseram-se que tenham caráter universal.
Assumimos esse pressuposto de taxonomia contingentes, mas para ter um ponto de partida de estudo, adotamos uma síntese das taxonomias (Borges et al., 2015), com quatro categorias: 1 . Condições contratuais e jurídicas, que se referem aos aspectos jurídicos (autônomo versus emprego), contratuais e de estabilidade no caso do emprego, além de abranger também o sistema de incentivo e a jornada de trabalho; 2 . Condições físicas e materiais, relativas a aspectos do entorno do trabalho como espaço arquitetônico e instalações, segurança física e/ou material e a formas de lidar com o impacto do espaço geográfico e condições climáticas; 3 . Processos e características da atividade, que abrangem os aspectos relativos ao conteúdo das atividades, à organização trabalho, às demandas e/ou requisitos do posto de trabalho, aos modos de realização das atividades e ao desempenho profissional; 4. Condições do ambiente sociogerencial, que se referem às interações interpessoais, às práticas sociais da gestão (no caso do emprego) e às práticas sociais decorrentes da inserção no mercado de trabalho (parcerias, redes de trabalho etc.).

Borges et al. (2013), desdobrando as categorias com base no questionário do European Working Conditions Observatory (EWCO), utilizado em estudos na União Europeia (European Foundation for the Improvement of Living and Working Conditions, http://www.eurofound. ie), desenvolveram o QCT, em português. Exploraram, então, tal síntese taxonômica com duas amostras: uma de 411 operários da construção de edificação e outra de 264 participantes trabalhadores de saúde e docentes de um centro universitário privado. Tertuliano e Borges (2019) focalizaram trabalhadores do saneamento básico $(n=453)$. Nesses casos, o tamanho das amostras não permitiu explorarem se as três categoriais tinham validade de fatores secundários. A extensão do quadro de pessoal da UFMG viabilizou formar amostra suficiente para tanto. Assim, definimos como primeiro objetivo específico: $\left.1^{\circ}\right)$ Verificar se o conjunto de itens que formam três categorias - condições físicas e materiais, processos e características da atividade, ambiente sociogerencial - agrupam-se em três fatores empíricos correspondentes.

Borges et al. (2013) e Tertuliano e Borges (2019) exploraram a estrutura fatorial dos itens em cada uma dessas três categorias de condições de trabalho e encontraram soluções distintas por amostras para elas, as quais contam com medidas escalares contínuas. Tal fatoração ofereceu sustentação empírica às tipologias concebidas a partir da reflexão teórica e das evidências empíricas anteriores. Porém, há heterogeneidade de consistência entre os fatores, como mencionado.

A amostra de docentes e profissionais de saúde (Borges et al., 2013) é a de características mais próximas ao segmento populacional em foco na presente pesquisa. Para aquela amostra, sete fatores têm coeficientes alfa de Cronbach entre 0,58 a 0,75, sendo 
eles: Espaço de Trabalho (Percepção de exposição a realizar as atividades nas instalações da organização, em campo e/ou no espaço virtual) e Aspectos FísicoQuímicos (Percepção de exposição a aspectos, como: vapores, fumaça e poeira; variações de temperatura e iluminação) na categoria das condições físicas e materiais; Complexidade, Responsabilidade e Rapidez, (Percepção das complexidade das tarefas, das exigências de rapidez, responsabilidade por danos e iniciativa diante do imprevisto), Organização do Tempo (Percepção da autonomia para organizar o próprio trabalho no tempo, planejar intervalos, folgas e férias) e Estímulo à Colaboração (Percepção da disponibilidade de colaboração na realização do trabalho) na categoria dos processos e características da atividade; Discriminação Social (percepção do participante quanto o gerenciamento na organização minimiza ações discriminatórias) e Participação (Percepção dos participantes sobre as práticas interativas de consulta na organização do trabalho e de abertura ao diálogo em torno do desempenho) na categoria das condições do ambiente sociogerencial. Tertuliano e Borges (2019) encontraram melhor consistência (coeficientes alfas de Cronbach de 0,66 a 0,86) nos fatores do QCT e atribuíram tais resultados a homogeneidade na amostra de trabalhadores operativos do saneamento básico.

A resolução no 09/2018 do Conselho Federal de Psicologia (CFP) classificou os fatores dos instrumentos psicológicos para uso prático segundo a consistência, como: suficientes, quando contam com coeficiente alfa de Cronbach a partir de 0,60; bons, a partir de 0,70; e excelentes, a partir de 0,80 . Abaixo do nível de consistência suficiente, o QCT apresentou um único fator para a amostra de docentes e profissionais de saúde: Discriminação Social $(\alpha=0,58)$. Entretanto, Cea D’Ancona (2004) recomendou só incluir em análises estatísticas, como análise de regressão e path analysis, escores das medidas com esse coeficiente $\geq 0,80$.

As demandas de aperfeiçoamento do QCT também dizem respeito à composição fatorial, se comparamos os fatores encontrados à dimensionalidade (Borges et al., 2015) explorada previamente à sua adaptação. Assim, em referência às condições físicas e materiais falta um fator abrangendo somente aspectos da adequação das instalações físicas, dos equipamentos e instrumentos de trabalho às atividades realizadas. Em referência aos processos e características da atividade, os itens indicadores do ritmo de trabalho e do seu conteúdo se reuniram em um único fator: Complexidade, Responsabilidade e Rapidez (alfa-0,75). Maslach e Leiter (1997/1999) afirmaram, por exemplo, que a carga de trabalho inclui o que o trabalhador faz e como faz, além de abranger intensidade, tempo gasto e complexidade. Portanto, o fator da maneira encontrada faz sentido. Mas não eliminamos a dúvida: tal composição fatorial seria um artefato dos itens utilizados? Poderíamos ter dois fatores? Ainda sobre os processos e características da atividade, a análise fatorial não identificou um fator referente ao papel social das atividades como previsto (Borges et al., 2015).

No que se referem às condições do ambiente sociogerencial, os fatores encontrados foram mais numerosos e, por consequência, mais focalizados. A fragilidade de consistência de dois fatores - Discriminação Social $(\alpha=0,58)$ e Participação $(\alpha=0,63)$-, entretanto, autoriza algumas indagações. Os itens do fator de Discriminação Social demandam outro formato mais sensível às manifestações dos preconceitos sutis ou encobertos (Fiske, 2017; Lins et al., 2014; Monroe \& Plant, 2019; Pereira \& Souza, 2016; Verniers \& Vala, 2018)? Os itens utilizados os operacionalizam adequadamente? Categorizações sociais (como ser produtivo ou não, ser TAE ou docente, ser efetivo ou não etc.) podem significar fontes de preconceitos para servidores. Sobre o fator Participação, indagamos se deveríamos abranger características das universidades federais, como as decisões colegiadas, o acesso à informação e a influência nos processos decisórios articulados às coalizações internas.

Todas essas dúvidas e/ou indagações sobre o QCT, na sua utilização para servidores de universidades públicas, as mudanças e a diversidade das atividades universitárias nos conduziram a definir o segundo e terceiro objetivos específicos, a saber: $2^{\circ}$ ) Implementar modificações no QCT visando a superação das limitações já comentadas e explorar empiricamente as estruturas fatoriais por categorias taxonômicas, avaliando seu aperfeiçoamento psicométrico; e $3^{\circ}$ ) Explorar a diferenciação da estrutura fatorial dos itens.

Esses dois objetivos exigem-nos revisar o QCT, considerando as singularidades das universidades federais. Segundo literatura consultada (p. ex., Leite, 2017; Santos et al., 2016), a docência tem uma dimensão burocrática-administrativa, além de que é central no trabalho dos TAEs. Para Santos et al., (2016), essa dimensão tem se expandido em contexto marcado pela lógica empresarial, em que compete ao docente buscar recursos financeiros em agências de fomento, gerenciando suas próprias condições de trabalho. Os modos de avaliação de produção ao docente também contribuíram para ampliar a referida dimensão. Leite acrescentou que o enxugamento do quadro de TAEs e a informatização dos registros sobrecarregaram os docentes com mais tarefas burocráticas. Em entrevistas com docentes (Rosa, 2017) e com TAEs (Macedo, 2018), há queixas acerca da racionalidade burocrática.

\section{Método}

Tendo em vista a consecução dos objetivos anunciados, desenvolvemos a revisão do QCT (etapa preparatória) e a pesquisa de campo como descreveremos na sequência. 


\section{Etapa Preparatória de Revisão do QCT}

Partimos da versão anterior do QCT (Borges et al., 2013) e de suas limitações descritas. Funcionamos como grupo de discussão, composto pelos autores, funcionários da UFMG e estudantes de pós-graduação (alguns professores de instituições particulares de ensino superior) e de graduação do curso de Psicologia da UFMG. Fizemos reuniões periódicas de maio/2016 a dezembro/2016. Discutimos acerca dos servidores da universidade e suas condições de trabalho, com base na literatura especializada, na experiência vivencial com a instituição e nos projetos de pesquisa em andamento no grupo de pesquisa. As discussões contribuíram adaptando itens e introduzindo outros que apreendessem singularidades das condições de trabalho nas universidades. Construímos uma nova versão do QCT, implementando modificações. Nas condições físicas e materiais, desdobramos alguns itens, bem como acrescentamos itens referentes às instalações (higiene, adequação e dimensionamentos) ao estado de conservação dos equipamentos, à disponibilidade de material de trabalho e de Equipamentos de Proteção Individuais (EPIs). Em processos e características da atividade, acrescentamos itens que permitissem o participante registrar suas percepções acerca do caráter burocrático-institucional, das oportunidades e necessidades contínuas de atualizações e requalificações, bem como do caráter planejado dos processos de trabalho. Nas condições do ambiente sociogerencial, acrescentamos itens sobre liberação e incentivo à qualificação, acesso a treinamento, apoio à socialização, aplicação do plano de carreira e participação nas decisões. Nos itens sobre discriminação, focalizamos o ambiente e não, exclusivamente, em si mesmo. Inspiramo-nos na literatura (Fiske, 2017; Lins et al. 2014; Monroe \& Plant, 2019; Pereira \& Souza, 2016; Verniers \& Vala, 2018) que aborda o conceito de preconceito sutil ou velado.

\section{Pesquisa de Campo}

\section{Participantes}

A amostra totalizou 1.060 participantes (13,68\% dos 7.748 servidores), dos quais $68 \%$ eram mulheres. Em referência ao estado civil, $49,6 \%$ se declararam solteiros, $30,0 \%$, casados e $20,4 \%$, outros. Em relação aos filhos, $54,2 \%$ da amostra têm ao menos um filho. A amostra apresentou idade média de 41,61 anos $(D P=10,36)$. Relativo ao cargo exercido na UFMG, 25,4\% dos participantes eram docentes de nível superior, $7,2 \%$, docentes de ensino fundamental e/ou médio e $67,4 \%$, TAEs. Do total de servidores UFMG, no final de 2017, $57,2 \%$ dos funcionários eram TAEs e $42,8 \%$, docentes (UFMG, 2018).

\section{Instrumentos Aplicados}

O instrumento principal aplicado foi a nova versão do QCT, construída na etapa preparatória, em formato impresso e online. Para esse último formato, contamos com serviço de programação especializado, garantindo a segurança dos dados, o sigilo, o anonimato dos participantes, a continuidade do preenchimento após interrupção e o impedimento de que o participante avançasse com itens em branco. A nova versão do QCT abrangeu 28 itens sobre as condições contratuais e jurídicas, 47 sobre as condições físicas e materiais, 74 sobre processo e características da atividade e 79 acerca das condições do ambiente sociogerencial. Os itens sobre as condições contratuais e jurídicas estruturam-se com alternativas nominais para as respostas, as quais não serão objeto de análise do presente artigo. Os demais usam escala com as seguintes alternativas: 1 (Nunca), 2 (Raramente), 3 (Algumas vezes), 4 (Muitas vezes), 5 (Todo o tempo) e 6 (Não se aplica). Aplicamos também uma ficha sociodemográfica e ocupacional, cujas respostas utilizamos para descrever a amostra.

\section{Procedimentos de Coleta de Dados}

Aplicamos os questionários, no ano de 2017, utilizando o sistema de informática da UFMG. No hospital, o QCT também foi aplicado no formato impresso para quem preferiu. Ambos os formatos eram precedidos por uma mensagem ao servidor, esclarecendo os objetivos da pesquisa, o caráter voluntário e anônimo da participação, bem como pelo Termo de Consentimento Livre e Esclarecido (TCLE).

\section{Registro e Procedimentos de Análise dos Dados}

A aplicação online implica uma tabulação automática em que recebíamos banco de dados do software "Excel" que, por sua vez, dialoga com o Statistical Package of Social Science (SPSS). Aqueles aplicados no formato impresso demandaram digitação manual diretamente no banco de dados do SPSS. Concluída a preparação do referido banco de dados, procedemos as análises estatísticas das respostas nas seguintes etapas:

\section{Análises Preliminares dos Itens do QCT}

Essas análises abrangeram a avaliação da normalidade, da fatorabilidade, da multicolinearilidade e de itens singulares (ou outliers). Para a avaliação da normalidade dos itens, usamos os coeficientes Skewness e Kurtosis (Cea D'Ancona, 2004). Seguimos a recomendação de Kim (2013) e Damásio (2012) de aceitar Skewness <2,0 e Kurtosis $<7,0$. Adicionalmente, explorarmos os gráficos de distribuição de frequência item a item. Excluímos, então, 24 itens ${ }^{2}$, cujas distribuições tendiam a concentração 
de escores em um dos extremos, aproximando-se do que sejam as constantes.

Conforme os objetivos da pesquisa, exploramos a estrutura fatorial com dois níveis: fatores de primeira e segunda ordem. Partimos da identificação dos fatores de segunda ordem. Assim, a exploração de multicolinearidade e de singularidades estatísticas ocorreu em cada conjunto de itens agregados pelos fatores secundários, considerando os coeficientes communalities e de correlações entre os itens.

Para a definição do número de fatores, consideramos o eigenvalue $\geq 1$, o gráfico screeplot e a interpretação dos fatores (uso combinado sugerido por Damásio, 2012). Como adotamos o conjunto de critérios citados, em todas as soluções os coeficientes eigenvalues mínimos variaram. Assim, o menor eigenvalue na estrutura de segunda ordem foi 10,36 e nas estruturas primárias foram: 1,85 (na primeira), 1,79 (na segunda) e 1,49 (na terceira).

\section{Análises Fatoriais Exploratórias (AFEs)}

Aplicamos AFE (Principal Axis Factoring) no conjunto das respostas aos itens do QCT das três categorias com questões escalares (condições físicas e materiais, processos e características da atividade e condições do ambiente sociogerencial) para explorar a diferenciação das categorias adotadas conceitualmente em pesquisas anteriores (ver objetivo específico 1). Consideramos os três fatores encontrados como de segunda ordem e fizemos novas AFEs no conjunto de itens que formavam cada fator secundário, encontrando os primários. Nas AFEs, aplicávamos rotação oblíqua e examinávamos se na matriz de correlação fatorial havia algum coeficiente acima de 0,30 . Nas três soluções fatoriais primárias, a rotação oblíqua foi confirmada, de modo que só adotamos a rotação Varimax na solução fatorial de segunda ordem.

\section{Resultados}

Da AFE com todos os itens do questionário, encontramos três conjuntos de itens, mas que não corresponderam àquelas três categorias - condições físicas e materiais, processos e caraterísticas da atividade e condições do ambiente sociogerencial. Por isso, designamos os fatores de segunda ordem de: 1. Exigências, Contradições e Condições Materiais; 2. Comunicação, Desafio e Participação; e 3. Suporte e Planejamento. Representamos a estrutura fatorial com os fatores de primeira e segunda ordem na Figura 1, que também mostra a proporção da explicação da variância e os coeficientes alfa de Cronbach de cada fator.

O primeiro fator de segunda ordem agrupou itens de todas as três categorias preliminares, sendo que os itens que se referiam a Condições Físicas e Materiais estão quase todos nesse fator, mas não predominaram entre os de cargas mais elevadas. As cargas em tal fator variaram de 0,32 a 0,70 . No intervalo de cargas de 0,60 a 0,70, há apenas dois itens originalmente de condições físicas e materiais (referentes a falta de materiais e riscos de pequenos acidentes). Provavelmente, isso significa que os participantes não as perceberam separadas das iniciativas de gestão e dos processos de trabalho, mas que as condições do ambiente físico os afetam intermediados por tais ações. Em contrapartida, os três itens de maiores cargas $(0,70,0,63$ e 0,61 respectivamente) se referem a: exigências desproporcionais às condições de trabalho; realizar tarefas conflitivas ou contraditórias e adoção de prazos incompatíveis às tarefas. Desses aspectos, derivamos a denominação do fator.

A natureza dos fatores primários, formados pelos itens que compõem o fator secundário em tela, clareia o que seja seu conteúdo (Tabela 1). O primeiro fator - Processo Burocrático - abarcou itens oriundos das inclusões realizadas na etapa de revisão do QCT. O segundo fator - Discriminação - abarcou itens predominantemente originários da categoria das condições do ambiente sociogerencial. Os itens referentes à exclusão (diminuição de oportunidades) predominaram entre os itens com cargas fatoriais maiores (de 0,65 a 0,82) em relação aos itens referentes a preconceitos expressos em insinuações. O terceiro fator - Ambiente Físico - reuniu itens originários das condições físicas e materiais. No quarto fator - Exigências - predominaram itens provenientes da categoria dos processos e características da atividade. No quinto fator - Violência - estão itens originários também na categoria do ambiente sociogerencial. No sexto fator - Respeito às Limitações - estão itens da mesma origem, mas que se referem ao respeito pelos tratamentos de saúde, pelos afastamentos, necessidades de pausa e limitações de cada servidor. O último fator - Precariedade Material - abarca três itens originários na categoria do ambiente sociogerencial e um de condições físicas e materiais (o de menor carga). Sobre os resultados dessas AFE, atentamos que os fatores Ambiente Físico, Exigências e Violências são correlacionados (Figura 1).

O segundo fator de segunda ordem - Comunicação, Desafio e Participação - apresenta predominância de itens originários na categoria de processos e características da atividade, de modo que focaliza parcialmente a organização ou o conteúdo do trabalho. Dizemos parcialmente porque há aspectos da organização do trabalho também nos demais fatores secundários. Sua estrutura primária conta com cinco fatores: Atualização do Saber, Responsabilidade, Participação, Informatização e Flexibilidade de Jornada. Resumimos a composição por itens na Tabela 2 , enquanto a proporção da explicação da variância, a consistência e correlação entre o primeiro e quinto fator, na Figura 1. 
Figura 1

Estrutura Fatorial de Primeira e Segunda Ordem do QCT

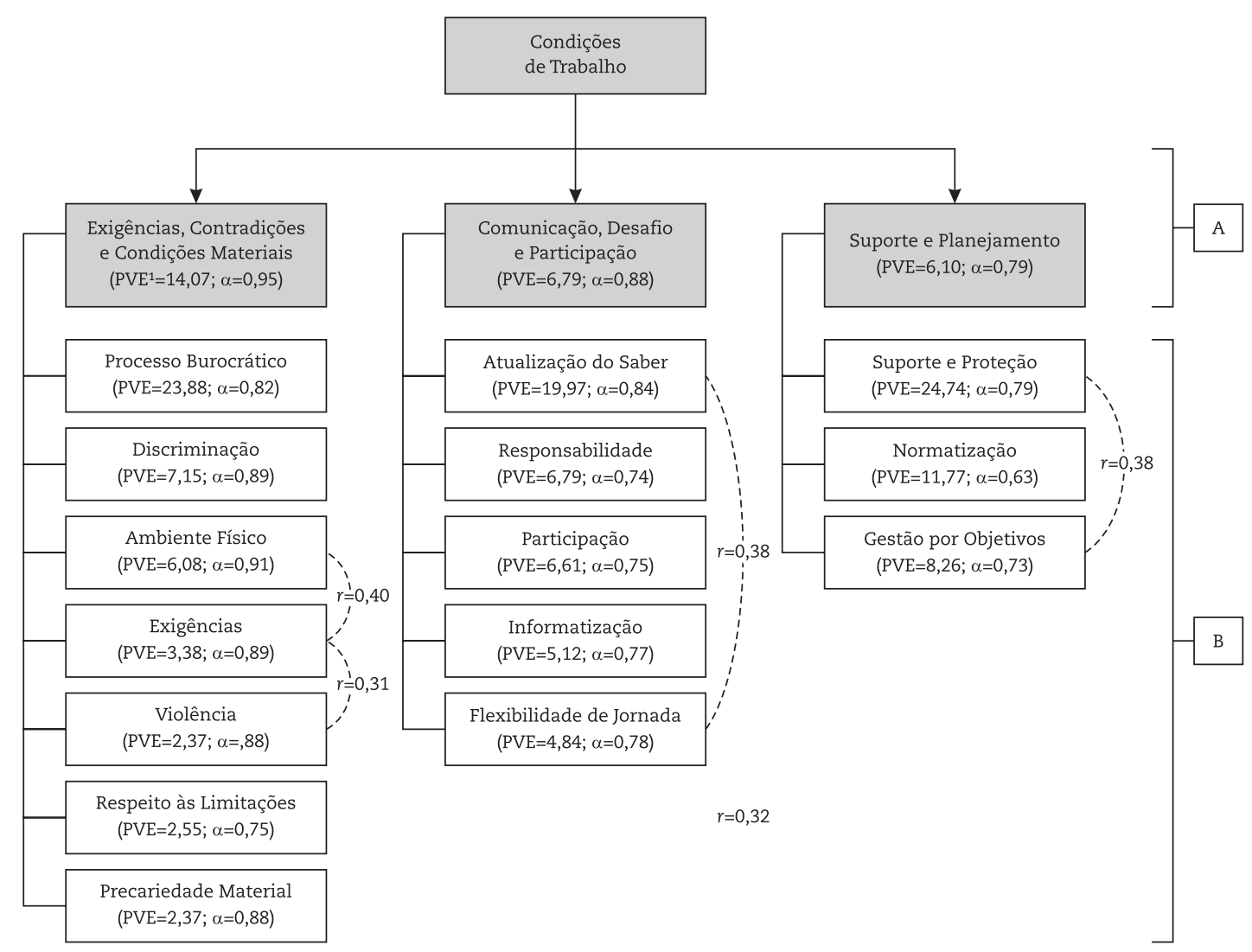

Legenda. ${ }^{1} \mathrm{PVE}=$ Proporção da variância explicada; Linhas representam correlações superiores a 0,30 entre fatores primários; $A=$ estrutura fatorial de segunda ordem; $B=e s t r u t u r a$ fatorial de primeira ordem

Tabela 1

Fatores de Exigências, Contradições e Condições Materiais (ECM)

\begin{tabular}{l} 
Fatores, Descrição e itens por carga \\
\hline Processo Burocrático \\
Descreve o caráter burocrático dos processos de trabalho, como cumprimento de normas, prescrição de tarefas, \\
e preenchimento de formulários abrangendo aspectos de racionalidade desconhecida. \\
Itens (carga): PC28(0,56); PC27(0,56); PC614(0,48); PC104(0,37) e PC613(0,36). \\
Discriminação \\
Refere-se à observação no ambiente de trabalho de exclusão e/ou diminuição de oportunidades por \\
características pessoais, religião, nacionalidade e regiões, deficiências físicas, idade e posicionamento político, \\
bem como de insinuações pelas razões anteriores e pelo setor de trabalho e vínculo empregatício. \\
Itens (carga): AG89(0,82); AG86(0,76); AG82(0,75); AG810(0,72); AG81(0,69); AG88(0,65); AG79(0,57); AG83(0,53); \\
AG71(0,50); AG78(0,47); AG87(0,46) e AG73(0,43).
\end{tabular}

\section{Ambiente Físico}

Refere-se à exposição a riscos (p. ex., produtos químicos, pequenos acidentes) e incômodo do ambiente natural (p. ex., ventilação inadequada, iluminação insuficiente, calor) e das instalações utilizadas (p. ex., falta de higiene no ambiente de trabalho, posições dolorosas ou fatigantes).

Itens (carga): FM11(0,70); FM12(0,67); FM23(0,66); FM19(0,65); FM9(0,61); FM28(0,58); FM30(0,57); FM10(0,57); FM1(0,56); FM6(0,56); FM17(0,53); FM33(0,52); FM2(0,52); FM8(0,51); FM35(0,51); FM26(0,50); FM32(0,48); FM22(0,45); $\operatorname{FM} 18(0,42) ; \operatorname{FM} 21(0,40)$ e FM3 $(0,40)$.

\section{Exigências}

Percebe as exigências para realização das tarefas, como ritmo acelerado, rigidez dos prazos, sobrecarga, incompatibilidades entre demandas de trabalho e número de servidores, cumprimento de metas de produção e cobranças desproporcionais.

Itens (carga): PC21(-0,76); PC22(-0,74); AG51(-0,66); PC23(-0,63); AG511(-0,62); PC25(-0,56); PC24(-0,52); PC49(-0,48); PC44(-0,43) e AG56 $(-0,37)$. 
Tabela 1 (continuação)

Fatores de Exigências, Contradições e Condições Materiais (ECM)

Fatores, Descrição e itens por carga

\section{Violência}

Diz respeito a observações de assédios morais, intimidações, agressões verbais, elogios inadequados, ameaças e outras formas de violência interpessoal.

Itens (carga): AG610(0,61); AG68(0,60); AG58(0,53); AG61(0,50); AG59(0,48); AG57(0,47); AG63(0,46); PC106(0,42); AG47(0,40); PC105(0,39); AG69(0,39) e PC107(0,37).

\section{Respeito às Limitações}

Referem-se ao respeito pelos tratamentos de saúde, pelos afastamentos, necessidades de pausa e limitações em geral de cada servidor.

Itens (carga): AG413(0,63); AG415(0,56); PC69(0,52); PC64(0,47) e AG414(-0,38).

\section{Precariedade Material}

Consideram à carência de equipamentos, de materiais, de softwares, entre outros, para a realização das tarefas. Itens (carga): AG53(0,73); AG52(0,72); AG54(0,69) e FM43(0,46).

Legenda. FM=Itens originários da parte do QCT dedicado às Condições Físicas e Materiais; PC=Itens originários da parte do QCT dedicado aos Processos e Características da Atividade; AG=Itens originários da parte do QCT dedicado às Condições do Ambiente Sociogerencial

\section{Tabela 2}

Fatores de Comunicação, Desafio e Participação

\section{Fatores, Descrição e itens por carga}

\section{Atualização do Saber}

Indica que as atividades dos servidores implicam a necessidade de atualização contínua de seus saberes e competências, formações complementares, domínio do saber-fazer e iniciativas de formação e informação que ultrapassam os limites organizacionais incluindo, por vezes, a necessidade de trabalhar em casa. Itens (carga): PC86(0,80); PC87(0,72); PC610(0,51); PC85(0,50); FM36(0,50); AG25(0,47); PC35(0,46); PC82(0,46); AG26(0,45); FM42(0,38); FM37(0,34) e AG52(0,33).

\section{Responsabilidade}

Refere-se à guarda e/ou preservação de documentos, de equipamentos, de informações e de sanções. Itens (carga): PC97(0,71); PC94(0,67); PC96(0,65); PC95(0,53); PC91(0,42) e AG514(0,33)

\section{Participação}

Diz respeito à capacidade de administrar os conflitos no ambiente de trabalho, ao dever de tomar parte nas decisões e à qualidade das decisões ponderadas coletivamente.

Itens (carga): AG45(0,69); AG44(0,66) e AG46(0,59).

\section{Informatização}

Refere-se ao uso de recursos de informática e de mídias nas comunicações no ambiente de trabalho. Itens (carga): PC85(-0,34); PC84(-0,76); PC83(-0,68) e PC410(-0,50).

\section{Flexibilidade da Jornada}

Abrange a ideia de autonomia em estabelecer horário, planejar as atividades, de mobilidade, porém com limitações de custeio.

Itens (carga): FM36(0,37); AG25(0,43); FM37(0,31); PC95(0,31); PC12(0,73); PC13(0,56); PC74(0,40); AG14(0,39); PC14(0,37); AG11(0,35) e AG31(0,34).

Legenda. FM=Itens originários da parte do QCT dedicado às Condições Físicas e Materiais; PC=Itens originários da parte do QCT dedicado aos Processos e Características da Atividade; AG=Itens originários da parte do QCT dedicado às Condições do Ambiente Sociogerencial

O terceiro fator de segunda ordem - Suporte e Planejamento - combina doze itens originários na categoria do ambiente sociogerencial e oito na de processos e características da atividade. O conjunto de seus itens aborda a gestão de segurança por ações de orientação e padronização, bem como a gestão das atividades centrada nas suas finalidades. Sua estrutura fatorial de primeira ordem é a mais simples, com três fatores - Suporte e Proteção, Normatização e Gestão por Objetivos - cujas composições dos itens sintetizamos na Tabela 3. A Figura 1 registra a correlação entre o primeiro e o último fator. 
Tabela 3

Suporte e Planejamento

Fatores, Descrição e itens por carga

Suporte e Proteção

Refere-se a ações de orientação e treinamento/reciclagem para evitar riscos de adoecimento e de acidentes no trabalho, bem como ao apoio dos chefes/superiores nas devolutivas sobre seu desempenho no trabalho.

Itens (carga): AG412(0,77); AG411(0,76); AG23(0,54); AG22(0,50); AG410(0,40); AG15(0,37); PC62(0,36); AG45(0,33) e AG43(0,33).

\section{Normatização}

Diz respeito à disponibilização de normas e padronizações (p. ex., manuais, regimentos, protocolos), bem como ações de chefes/superiores que norteiam e limitam a realização de tarefas. Itens (carga): PC71(0,61); PC42(0,54); PC72(0,43) e PC93(0,37).

\section{Gestão por Objetivos}

Indica o quanto a realização das atividades se norteia pelos objetivos estabelecidos, pelo planejamento realizado, pelo clima de diálogo e administração de conflito nas relações interpessoais e entre setores. Itens (carga): PC103(0,70); PC108(0,68); PC101(0,50); AG61(-0,45); AG45(0,44); AG43(0,41) e AG73(-0,31).

Legenda. FM=Itens originários da parte do QCT dedicado às Condições Físicas e Materiais; PC=Itens originários da parte do QCT dedicado aos Processos e Características da Atividade; AG=Itens originários da parte do QCT dedicado às Condições do Ambiente Sociogerencial

\section{Discussão}

Atingimos o primeiro objetivo específico da presente pesquisa - Verificar se o conjunto de itens que formam três categorias (condições físicas e materiais, processos e características da atividade; ambiente sociogerencial) se agrupam em três fatores correspondentes - pela aplicação da análise fatorial exploratória em que identificamos os fatores de segunda ordem. Os resultados mostraram que, no caso dos servidores da UFMG da amostra, aquelas categorias teóricas das pesquisas anteriores não organizavam suas percepções acerca das condições de trabalho. Isso representa uma passagem de um conhecimento abstrato para um mais empírico, sustentado na realidade e/ou nas concepções sobre condições de trabalho derivadas das vivências concretas dos servidores. Fortalece a construção de uma psicologia concreta (Lima, 2002). É, portanto, a primeira contribuição científica desta pesquisa. Em contrapartida, ponderamos que os fatores de segunda ordem encontrados não podem ser generalizados para outras categorias ocupacionais. Lembramos do caráter contingencial das condições de trabalho e da percepção dos trabalhadores sobre elas. Por um lado, podemos compreender essa restrição como uma limitação da pesquisa. Por outro, ela corrobora a literatura consultada (Álvaro \& Garrido, 2006; Blanch, 2003; Ramos et al., 2002) no que se refere a se opor a uma classificação universal. Álvaro (1995) já afirmara que a tendência na Psicologia Social seria deixar de verificar teoria e passar a compreender a explicação alcançada em cada contexto.

Os três fatores de segunda ordem podem ser compreendidos como eixos organizadores da percepção dos participantes em relação às condições de trabalho vivenciadas. $\mathrm{O}$ primeiro fator de segunda ordem abriga fatores primários que refletem incômodos e/ou sofrimentos dos participantes sendo o único contraponto o fator de Respeito às Limitações. Uma hipótese explicativa para esse fato é que os impedimentos de quem gerencia para superar as situações incômodas, seja por disponibilidades de recursos, seja por aspectos culturais e de sobrecarga, têm sido compensados nas relações interpessoais pelo Respeito às Limitações de cada um. Como a literatura consultada (p. ex., Ramos \& Macêdo, 2018; Ruza \& Silva, 2016; Santos et al., 2016) apontou prevalências de alterações de saúde em servidores públicos, compete-nos sugerir que pesquisas futuras explorem se um desequilíbrio nestes fatores são aspectos contribuintes. Esse fator secundário na sua composição também reflete a contradição do ambiente universitário de ser exigente ou meritocrático a despeito de condições reais (p. ex., burocratização e precariedade material). Igualmente sugerimos explorar se tal contradição, que o QCT passou a dar conta de apreender, seria preditor de saúde/adoecimento mental.

O segundo fator de segunda ordem agrega fatores primários que têm em comum a tendência de o contexto universitário valorizar o servidor pela natureza de sua inserção ativa nos processos de trabalho, participando de decisões, vivenciando conflitos interpessoais, assumindo responsabilidades e percebendo sua qualificação como parte significativa dos processos produtivos. A abrangência de conteúdo desse fator de segunda ordem e sua respectiva estrutura fatorial primária indicam que o QCT está, em certa medida, apreendendo as relações de poder da estrutura organizacional predominantemente colegiada. Tendo em vista pesquisas futuras, indagamos: qual a potência desses fatores em promover saúde? O terceiro fator de segunda ordem combina fatores que têm em comum contrapartidas intangíveis na forma de suporte, 
orientações normativas e acesso e domínio dos objetivos institucionais. Outras pesquisas podem explorar o papel desses fatores na promoção de saúde.

Tal estrutura de segunda ordem se, de um lado não corroborou as categorias inicialmente previstas, de outro, contribuiu para a consecução do segundo objetivo específico (Implementar modificações no QCT visando a superação das limitações comentadas e explorar empiricamente as estruturas fatoriais por categoria taxonômicas, avaliando seu aperfeiçoamento psicométrico). Esse objetivo tem em parte um caráter metodológico. Assim, sua consecução se iniciou com os esforços de reconstrução do QCT. Esses esforços se refletiram nos resultados das AFEs. Permitiram a apreensão de fatores de segunda ordem contingentes à amostra de servidores de universidade. Os coeficientes alfas de Cronbach fortaleceram-se, já que na estrutura fatorial (Figura 1) temos 15 fatores primários dos quais: sete apresentaram nível de excelência; sete, nível bom; e apenas um, de suficiência. Não tivemos nenhum desses coeficientes menor que 0,60.

Os resultados das AFEs para essa versão revisada do QCT mostraram avanços na direção do terceiro objetivo específico, o qual visava mais ajuste às singularidades das condições de trabalho de servidores das universidades federais. A primeira contribuição está no conjunto da estrutura que, além de escapar à categorização geral inicial, identificou em cada estrutura primária de fatores como aqueles que mais explica a variância: Processo Burocrático, Atualização do Saber e Suporte e Proteção. Além disso, alguns fatores são correlacionados: 1. Ambiente Físico com Exigências e este com Violência, indicando que o ambiente físico desfavorável e as vivências de violência tornam as exigências mais desproporcionais, bem como essas podem ser tomadas como um tipo de violência; 2. Atualização do Saber com Flexibilidade de Jornada, indicando que a oportunidades de atualização e melhoria da qualificação para serem viabilizadas necessitam contar com a gestão que mantém a flexibilidade de horários; 3. Suporte e Proteção com Objetivos, indicando que a clareza e domínio dos objetivos fortalecem a percepção de suporte e proteção. Sugerimos que pesquisas futuras explorem os sentidos de tais correlações.

O terceiro objetivo específico também abarcava as intenções de aperfeiçoar a composição dos fatores. Elas se cumpriram nos seguintes aspectos dos resultados:

1. Identificamos um fator primário específico acerca do Processo Burocrático, que a literatura (Leite, 2017; Macedo, 2018; Rosa, 2017; Santos et al., 2016) levava a crer na sua importância.

2. Fortalecemos o fator de Participação, cujo coeficiente alfa melhorou (de 0,63 para 0,75 ) embora siga demandando aperfeiçoamento, que incluiu tomar parte em decisões colegiadas e perceber-se como agente de mudanças, bem como as vivências de conflitos.

3. Fortalecemos o fator Discriminação (de $\alpha=0,58$ passou a $\alpha=0,89$ ) e abarcou não só as categorias societais de discriminação social (como raça e gênero), mas também aspectos do ambiente acadêmico (como setor e atividade, posicionamentos políticos e vínculo contratual). Essa melhoria corroborou o acerto de focalizarmos a redação dos itens no ambiente;

4. Obtivemos um fator específico de Responsabilidade, refletindo os itens acrescentados e modificados na parte do QCT referente aos processos e características da atividade;

5. Designamos de Exigências ao fator que reuniu itens referentes ao ritmo ou rapidez no trabalho, bem como sobre pressão por prazos e sobrecarga.

6. Não encontramos um fator específico sobre o papel social das atividades, mas o fator de segunda ordem designado de Suporte e Planejamento abarca um primário que focaliza os objetivos como guia das ações e do planejamento. Se o servidor é consciente e/ou se identifica aos objetivos institucionais suas ações cotidianas no trabalho ganha sentido.

7. Não encontramos um fator específico sobre condições das instalações físicas, equipamentos e instrumentos, desapontando as expectativas. Consideramos que os participantes da amostra não percebem tais aspectos das condições de trabalho como algo central ou destacado no exercício de suas atividades. Nas IFES, há a tradição de conceber o projeto, os objetivos e definir aonde se quer chegar para, só depois, buscar os meios disponíveis (editais de pesquisa, órgãos de fomento etc.) ou se criar novos meios.

Esses aspectos apreendidos em relação a consecução do terceiro objetivo específico contribuíram para dirimir as dúvidas acerca do QCT referidas introdutoriamente. Pelas considerações anteriores, concluímos que atingimos adequadamente os objetivos de pesquisa. Entendemos, assim, que as IFES contam com um QCT útil para diagnósticos de condições de trabalho. O caráter exploratório da pesquisa, no entanto, por si já reivindica a continuidade de pesquisas acerca das condições de trabalho para os servidores das universidades federais. Sugerimos que se explore as diferenças de escores entre docentes e TAEs, entre estatutários e celetistas, entre servidores em estágio probatório. Além disso, devemos registrar como limite da pesquisa a amostra apresentar uma proporção de servidores TAEs mais acentuada que a população. Na pesquisa atual, ocupamo-nos da validade de construto, identificando uma estrutura fatorial que permita apreender as percepções dos servidores das universidades e a consistência dos fatores componentes, mas em pesquisa futura seria uma contribuição importante explorar a validade preditiva em relação, por exemplo, a saúde mental e qualidade de vida, haja vista as menções ao adoecimento dos servidores.

\section{Agradecimentos}

Registramos que o presente artigo relata parcialmente os resultados de projeto de pesquiso financiado 
pelo CNPq na forma de bolsa de produtividade em pesquisa à primeira autora. Registra-se que a parceira entre os autores contou com o suporte do Programa de Pós-Graduação em Psicologia da UFMG.

\section{Financiamento}

Todas as fontes e financiamento para elaboração e produção do estudo foram fornecidas pelo projeto de pesquisa intitulado "Qualidade de vida, condições de trabalho de servidores do UFMG" sob financiamento do $\mathrm{CNPq}$, a título de bolsa de produtividade em pesquisa de primeira autora do artigo, conforme processo 311764/204-2.

\section{Contribuições dos autores}

Declaramos que todos os autores participaram da elaboração do manuscrito. Especificamente, a autora Livia de Oliveira Borges participou da redação inicial do estudo, conceitualização, investigação, visualização, o(s) autor(es) Livia de Oliveira Borges, Geraldo Majela Garcia Primo, Sabrina Cavalcanti barros, Silvino Paulino dos Santos Neto, Camila Teixeira Heleno e Georgina Maria Véras Motta participaram do desenvolvimento do método, atividades de campo e de análises dos dados, bem como, da redação final do trabalho, revisão e edição.

\section{Disponibilidade dos dados e materiais}

Todos os dados e sintaxes gerados e analisados durante esta pesquisa serão tratados com total sigilo devido às exigências do Comitê de Ética em Pesquisa em Seres Humanos. Porém, o conjunto de dados e sintaxes que apoiam as conclusões deste artigo estão disponíveis mediante razoável solicitação à autora principal do estudo.

\section{Conflito de interesses}

Os autores declaram que não há conflitos de interesses.

\section{Referências}

Álvaro, J. L. (1995). Psicología Social: Perspectivas Teóricas y Metodológicas. Siglo XXI de España Editores.

Álvaro, J. L., \& Garrido, A. (2006). Trabajo, ocupación y bienestar. Em A. Garrido (Org.), Sociopsicología del Trabajo (pp. 99-132). Editorial UOC.

Blanch, J. M. (2003). Condiciones de trabajo. Em J. M. Blanch, M. J. Espuny, C. Gala \& A. Martín (Orgs.), Teoría de las Relaciones Laborales. Fundamentos (pp. 42-44). Editorial UOC.

Borges, L. O., Costa, M. T. P., Alves-Filho, A., Souza, A. L. R., Rocha-Falcão, J. T., Leite, C. P. R. A., \& Barros, S. C. (2013). Questionário de condições de trabalho: reelaboração e validação de construto. Avaliação Psicológica, 12(2), 213-225. http://pepsic.bvsalud.org/scielo. php?script $=$ sci_arttext\&pid $=$ S1677-04712013000200012

Borges, L. O., Rocha-Falcão, J. T., Alves Filho, A., \& Costa, M. T. P. (2015). Condições de trabalho. Em P. F. Bendassolli \& J. E. BorgesAndrade. (Org.), Dicionário de Psicologia do Trabalho e das Organizações (pp. 227-236). Casa do Psicólogo.

Cea D’Ancona, A. (2004). Métodos de Encuesta. Teoría y Práctica, Errores y Mejora. Editorial Sintesis.

Conselho Federal de Psicologia (2018). Resolução Nº 9, de 25 de abril de 2018. http://satepsi.cfp.org.br/docs/Resolu\%C3\%A7\%C3\%A3oCFP-n\%C2\%BA-09-2018-com-anexo.pdf

Costa, M. T. P., Borges, L. O., \& Barros, S. C. (2015). Condições de trabalho e saúde psíquica: um estudo em dois hospitais universitários eletrônicos. Revista Psicologia: Organizações e Trabalho, 15(1), 43-58. http://dx.doi.org/10.17652/rpot/2015.1.490

Damásio, B. F. (2012). Uso da análise fatorial exploratória em psicologia. Avaliação Psicológica, 11(2), 213-228. http://pepsic.bvsalud.org/pdf/ avp/v11n2/v11n2a07.pdf

Fiske, S. T. (2017). Prejudices in cultural contexts: shared stereotypes (gender, age) versus variable stereotypes (race, ethnicity, religion). Perspective on Psychological Science, 12(5), 791-799. https://doi.org/10.1177/1745691617708204

Forattini, C. D., \& Lucena, C. (2015). Adoecimento e sofrimento docente na perspectiva da precarização do trabalho. Laplage em Revista, 1(2), 32-47. https://doi.org/10.24115/S2446-622020217Extra-D1140p.575-581

García López, V. (2017). III Encuesta Navarra de Salud y Condiciones de Trabajo. Principales hallazgos. Archivos de Prevención de Riesgos Laborales, 20(2), 102-110. https://dx.doi.org/10.12961/aprl.2017.20.02.3.

Geraldini, J. R., \& Bicalho, P. P. G. (2016). Modos de subjetivação no sistema avaliativo da pós-graduação: conhecer, estranhar, interrogar. Revista Subjetividades, 16(1), 49-61. http://dx.doi.org/10.5020/23590777.16.1.78-90

Kim, H. Y. (2013). Statistical notes for clinical researchers: assessing normal distribution (2) using skewness and kurtosis. Restorative Dentistry E Endodontics, 38(1), 52-54. http://dx.doi.org/10.5395/rde.2013.38.1.52

Leite, J. L. (2017). Publicar ou perecer: a esfinge do produtivismo acadêmico. Revista Katalysis, 20(2), 207-215. https://doi.org/10.1590/1982$02592017 \mathrm{v} 20 \mathrm{n} 2 \mathrm{p} 207$

Lima, M. E. A. (2002). Esboço de uma Crítica à Especulação no Campo de Saúde Mental e Trabalho. Em M. G. Jacques, \& W. Codo (Orgs.), Saúde mental E trabalho: leituras (pp.50-81). Vozes.

Lins, S. L. B., Lima-Nunes, A., \& Camino, L. (2014). O papel dos valores sociais e variáveis psicossociais no preconceito racial brasileiro. Psicologia \& Sociedade, 26(1), 95-105. https://doi.org/10.1590/S0102-71822014000100011

Macedo, A. P. D. (2018). Remoções em estágio probatório e socialização organizacional: um estudo com os assistentes em administração de uma universidade federal [Dissertação de mestrado, Universidade Federal de Minhas Gerais]. Repositório Institucional da Universidade Federal de Minhas Gerais. http://hdl.handle.net/1843/BUOS-B6ZFYD 
Maia, L. C., Eira, R., Alves, A. C., \& Leão, C. P. (2015). The organizational improvement as trigger for better working conditions. RISTI Revista Ibérica de Sistemas e Tecnologias de Informação, (spe4), 50-65. https://doi.org/10.17013/risti.e4.50-65

Maslach, C., \& Leiter, M. P. (1999). Trabalho: Fonte de prazer ou desgaste? (M. S. Martins, trad.). Papirus. (Originalmente publicado em 1997)

Monroe, A. E., \& Plant, E. A. (2019). The dark side of morality: prioritizing sanctity over care motivates denial of mind and prejudice toward sexual outgroups. Journal of Experimental Psychology: General, 148(2), 342-360. https://doi.org/10.1037/xge0000537

Ordaz Castillo, E., \& Ronda-Pérez, E. (2015). Salud y condiciones de trabajo en trabajadores mayores. Medicina y Seguridad del Trabajo, 61(240), 314-324. https://dx.doi.org/10.4321/S0465-546X2015000300002.

Pasquali, L. (2003). Psicometria: Teoria dos testes na Psicologia e Educação. Vozes.

Pereira, C. R., \& Souza, L. E. C. (2016). Fatores Legitimadores da discriminação: uma revisão teórica. Psicologia: Teoria e Pesquisa, 32(2), 1-10. https://doi.org/10.1590/0102-3772e322222

Pietro, C. (1994). Trabajadores y Condiciones de Trabajo. Ediciones HOAC.

Pietro, C., Arnal, M., Caprile, M., \& Potrony, J. (2009). La Calidad del Empleo en España: Una Aproximación Teórica y Empírica. Ministerio del Trabajo e Inmigración.

Pizzio, A., \& Klein, K. (2015). Qualidade de vida no trabalho e adoecimento no cotidiano de docentes do Ensino Superior. Educação E Sociedade, 36(131), 493-513. http://www.scielo.br/pdf/es/v36n131/1678-4626-es-36-131-00493.pdf

Ramos, L. F. C., \& Macêdo, K. B. (2018). Reflexões sobre o adoecimento dos servidores técnico-administrativos em educação. Argumentum, 10(3), 107-122. https://doi.org/10.18315/argumentum.v10i3.16911

Ramos, J., Peiró, J. M., \& Ripoll, P. (2002). Condiciones de trabajo y clima organizacional. Em J. M. Peiró \& F. Prieto (Orgs.), Tratado de Psicología del Trabajo: la actividad laboral en su contexto (pp. 37-92). Síntesis Psicología.

Rosa, M. C. G. (2017). Necessidades e processos de tutorização organizacional: estudo com docentes e funcionários técnico-administrativos da UFMG [Dissertação de mestrado, Universidade Federal de Minas Gerais]. Repositório Institucional da Universidade Federal de Minas Gerais. https://repositorio.ufmg.br/handle/1843/BUBD-AQNFL3

Ruza, F. M., \& Silva, E. P. (2016). As transformações na pós-graduação: o prazer no trabalho docente está suspenso? Revista Subjetividades, 16(1), 91-103. http://dx.doi.org/10.5020/23590777.16.1.91-103

Santos, D. A. S., Azevedo, C. A., Araújo, T. M., \& Soares, J. F. S. (2016). Reflexões sobre a saúde docente no contexto de mercantilização do ensino superior. Revista de Docência do Ensino Superior, 6(1), 159-186. https://doi.org/10.35699/2237-5864.2016.2105

Souza, A. L. R. (2014). Condições de Trabalho e Comprometimento com a Organização e com a Carreira de Professores em um Centro Universitário [Tese de doutorado, Universidade Federal de Minas Gerais]. Repositório Institucional da Universidade Federal de Minas Gerais. https:// repositorio.ufmg.br/handle/1843/BUOS-9TNHCF

Tavares, J. P., Beck, C. L. C., Magnago,T. S. B. S., \& Lautert, L. (2012). Minor psychiatric disorders among nurses university faculties. Revista Latino-Americana de Enfermagem, 20(1), 175-182. https://doi.org/10.1590/S0104-11692012000100023

Tertuliano, M. V., \& Borges, L. O. (2019). O questionário de condições de trabalho para trabalhadores do saneamento básico. Revista Laborativa, 8(2), 57-78. https://ojs.unesp.br/index.php/rlaborativa/article/view/2877/pdf

Universidade Federal de Minas Gerais, UFMG (2018). Relatório de Gestão do Exercício de 2017. https://www.ufmg.br/proplan/wp-content/ uploads/UFMG_Relat\%C3\%B3rio-de-Gest\%C3\%A3o-2018.pdf

Verniers, C., \& Vala, J. (2018). Correction: justifying gender discrimination in the workplace: the mediating role of motherhood myths. Plos One, 13(7), e0201150. https://doi.org/10.1371/journal.pone.0201150

\section{Sobre os autores}

Livia de Oliveira Borges é Psicóloga (UFRN) e doutora em Psicologia pela Universidade de Brasília, com estágio pós-doutoral na Universidade Complutense de Madri. Professora titular aposentada atuando voluntariamente no Programa de Pós-graduação em Psicologia da UFMG e Bolsista de Produtividade em Pesquisa pelo CNPq.

Geraldo Majela Garcia Primo é Médico Sanitarista e do Trabalho. Doutor em Psicologia Social pela UFMG. Atua como funcionário técnico-administrativo no Hospital Universitário da UFMG.

Sabrina Cavalcanti Barros é psicóloga (UFSJ). Doutora em Psicologia Social pela Universidade Federal de Minas Gerais e pela Universidade Complutense de Madrid (cotutela). Professora na Faculdade de Ciências da Saúde da Região do Trairi (FACISA) da Universidade Federal do Rio Grande do Norte.

Silvino Paulino dos Santos Neto é graduado em Gestão de Recursos Humanos. Doutor em Psicologia Social pela Universidade Federal do Minas Gerais. Professor no Centro Mineiro de Ensino Superior Promove e nas Faculdades Kennedy.

Camila Teixeira Heleno é psicóloga e doutora em Psicologia pela UFMG com estágio doutoral na Universidade de Oviedo e pósdoutoral na UFMG. Professora na Universidade Federal dos Vales do Jequitinhonha e Mucuri.

Georgina Maria Véras Motta é psicóloga (PUC-MG), Doutora em Psicologia pela Universidade Federal do Minas Gerais, Membro da Coordenação do Fórum Sindical e Popular de Saúde e Segurança do Trabalhador de Minas Gerais.

\section{Como citar este artigo}

Borges, L. O., Primo, G. M. G., Barros, S. C., Santos Neto, S. P., \& Heleno, C. T., \& Motta, G. M. V. (2021). Questionário de Condições de Trabalho para Servidores Universitários. Avaliação Psicológica, 20(3), 379-389. http://dx.doi.org/10.15689/ap.2021.2003.19463.12 